\title{
Coupled Waves in Two-Phase Periodic Planar Multilayers
}

\author{
J. KAPELEWSKI*
}

Faculty of Electronics, Military University of Technology, S. Kaliskiego 2, 00-908 Warsaw, Poland

In the paper a consequently dynamic model approach to the magnetoelectric coupling is proposed. As opposed to the static case the approach includes both the elastic equation of motion and the full set of the Maxwell equations for effective composite medium. The analytical treatment is performed from two layers of $\infty_{m}$ symmetry and results in closed relations for propagation characteristics of coupled elasto-electrodynamic waves for millimeter-length range.

PACS: 41.20.Jb, 43.20.Mv, 03.50.De

\section{Introduction}

Multiferroic materials with coexistence of at least two ferroic orders (ferroelectric, ferromagnetic, or ferroelastic) have drawn increasing interest due to their potential for applications as multifunctional devices. In multiferroic materials, the coupling interaction between the different order parameters could produce new effects, such as magnetoelectric (ME) effect. The magnetoelectric response is the appearance of an electric polarization $\boldsymbol{P}$ upon applying a magnetic field $\boldsymbol{H}$ (i.e., the direct $\mathrm{ME}$ effect, designated as $\mathrm{ME}_{H}$ effect: $\left.\boldsymbol{P}=\alpha \boldsymbol{H}\right)$ and/or the appearance of a magnetization $\boldsymbol{M}$ upon applying an electric field $\boldsymbol{E}$ (i.e., the converse ME effect, or $\mathrm{ME}_{E}: \boldsymbol{M}=\alpha \boldsymbol{E}$ ).

Magnetoelectricity has been observed as an intrinsic effect in some natural material systems at low temperature, which have been under intensive study recently, motivated by potential applications in information storage, spintronics, and multiple-state memories. Although over ten different compound families have been widely investigated as multiferroic ME materials, a high inherent coupling between multiferroic order parameters (especially above room temperature) has not yet been found in a single-phase compound, which hinders their applications.

Alternatively and with greater design flexibility, multiferroic ME composites made by combining piezoelectric and magnetic substances together have drawn significant interest in recent years due to their multifunctionality, in which the coupling interaction between piezoelectric and magnetic substances could produce a large ME response (e.g., several orders of magnitude higher than that in those single-phase ME materials so far available) at room temperature. These ME composites provide opportunities for potential applications as multifunctional devices such as magnetoelectric transducers, miniaturized $\mathrm{mm}$

* e-mail: jerzy.kapelewski@wat.edu.pl and $\mu \mathrm{m}$ EM waves antennas, radomes, actuators, and sensors.

The ME effect in composite materials is known as a product tensor property, which results from the cross interaction between different orderings of the two phases in the composite. Neither the piezoelectric nor magnetic phase has the ME effect, but composites of these two phases have remarkable ME effect. Thus the ME effect is a result of the product of the magnetostrictive effect (magnetic/mechanical effect) in the magnetic phase and the piezoelectric effect (mechanical/electrical effect) in the piezoelectric one, namely,

$$
\begin{aligned}
& \mathrm{ME}_{H} \text { effect }=\frac{\text { magnetic }}{\text { mechanical }} \times \frac{\text { mechanical }}{\text { electric }}, \\
& \mathrm{ME}_{E} \text { effect }=\frac{\text { electric }}{\text { mechanical }} \times \frac{\text { mechanical }}{\text { magnetic }} .
\end{aligned}
$$

This is a coupled electrical and magnetic phenomenon via elastic interaction. That is, for the $\mathrm{ME}_{H}$ effect, when a magnetic field is applied to a composite, the magnetic phase changes its shape magnetostrictively. The strain is then passed along to the piezoelectric phase, resulting in an electric polarization. Thus, the ME effect in composites is extrinsic, depending on the composite microstructure and coupling interaction across magnetic-piezoelectric interfaces [1-3].

Thus, a new property (i.e., nonzero ME coefficient) appears in the composite consisting of magnetic and piezoelectric phases, since neither constituent phase is magnetoelectric. This new ME product response is due to elastic coupling between the two constituent phases. High piezomagnetic and piezoelectric coefficients and strong coupling favor a large ME coefficient.

Antenna miniaturisation has received significant attention in recent years because of demands for miniaturisation is more obvious for lower frequency bands where wavelength is larger. At low frequencies, such as $\mathrm{HF}$ and UHF, a standard half-wavelength dipole is about a few 
meters long, which is prohibitively large. There are several approaches to antenna miniaturisation, which can be classified into three categories. The first is to use capacitive or inductive loadings, and/or meandered lines to obtain allow-wave resonance, but increased second is to reduce the wavelength of the structure using dielectric materials, but is prone to surface-wave excitations and the corresponding losses of efficiency. The third approach is to use engineered ME materials for antenna miniaturisation [1].

In such slow-wave structures, the permeability of the material also contributes to the miniaturisation factor $n=\sqrt{\mu_{\mathrm{r}} \varepsilon_{\mathrm{r}}}$, and therefore smaller $\varepsilon_{\mathrm{r}}$ are required to achieve the same miniaturisation compared with pure dielectric loading. Another aim is to achieve $\mu_{\mathrm{r}}=\varepsilon_{\mathrm{r}}$. In this case, the characteristic impedance of the medium is equal to that of the free-space. As a result, no reflections take place between the substrate of the antenna and surrounding free space, thereby reducing the energy trapped in the substrate.

The foregoing reasons underscore the importance of possibly thorough understanding of mechanisms which can be effectively used for manipulating the effective materials parameters of ME multilayer. Some recent publications seem to indicate that an especially strong ME coupling can be produced in some phase transition critical regime (e.g. [4]).

In the paper, a simple analytical way to describe the elastically mediated magnetoelectric coupling in laminar two-phase composites is outlined. The main purpose of the analysis presented is to highlight the mechanism of the coupling as produced by the product effect on consequently dynamical footing. Both phases are modeled by alternately situated piezoelectric and piezomagnetic layers (Fig. 1). The resulting formula is instructive for clearly illustrating the essence of that kind of ME coupling in a possibly simple variety. The effect in consideration, can be regarded as useful basis for modeling effective electrodynamics (electric permittivity and magnetic permeability) and coupled parameters in ME planar composites.

The effect in consideration is known to be of particular interest for synthesis of various microwaves device such as filters, antenna, supports, radomes, frequency selective systems, sensors, etc.

\section{Description of the approach}

Each electric and magnetic layer of the planar composite in consideration provides a formal contribution to current sources called "polarization current" given by

$$
\begin{aligned}
& \boldsymbol{J}=\mathrm{j} \omega \varepsilon \boldsymbol{D} \theta_{p}(z), \\
& \boldsymbol{J}^{m}=\mathrm{j} \omega \mu \boldsymbol{B} \theta_{m}(z)=\boldsymbol{M},
\end{aligned}
$$

with $\theta_{p}(z)$ and $\theta_{m}(z)$ denoting $z$ cross-sections of material supports for the "currents" given in Eq. (1) electric and magnetic layers, respectively.

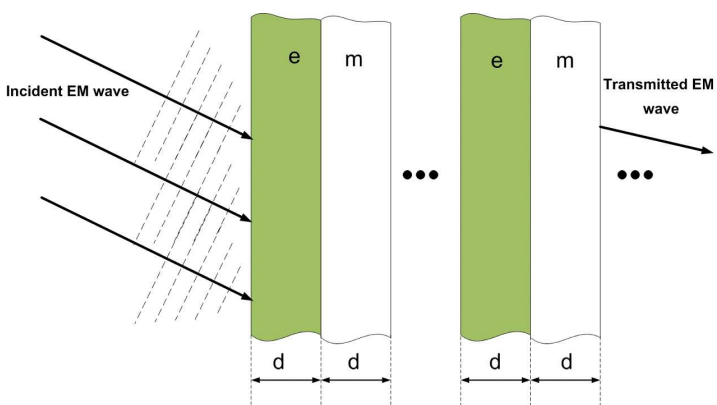

Fig. 1. The multiferroic superlattice composed of alternating ferroelectric and ferromagnetic layers.

For a monochromatic EM wave the full rotational part of the Maxwell equations can then be written in the form

$$
\begin{aligned}
& \boldsymbol{k} \times \boldsymbol{H}+\omega \boldsymbol{D}=\omega \delta \varepsilon \boldsymbol{E}, \\
& \boldsymbol{k} \times \boldsymbol{E}+\omega \boldsymbol{B}=\omega \delta \boldsymbol{\mu} \boldsymbol{H} .
\end{aligned}
$$

Effective constitutive relations for the superlattice (taken as a whole) are postulated in the following general form:

$$
\begin{aligned}
& \boldsymbol{T}^{\mathrm{e}}=\boldsymbol{C}^{\mathrm{t}} \boldsymbol{S}^{\mathrm{e}}-\boldsymbol{e}^{\mathrm{t}} \boldsymbol{E}^{\mathrm{e}}-\boldsymbol{q}^{\mathrm{t}} \boldsymbol{H}^{\mathrm{e}}, \\
& \boldsymbol{D}^{\mathrm{e}}=\boldsymbol{e}^{\mathrm{tT}} \boldsymbol{S}^{\mathrm{e}}+\boldsymbol{\varepsilon}^{\mathrm{t}} \boldsymbol{E}^{\mathrm{e}}+\boldsymbol{\alpha}^{\mathrm{t}} \boldsymbol{H}^{\mathrm{e}}, \\
& \boldsymbol{B}^{\mathrm{e}}=\boldsymbol{q}^{\mathrm{tT}} \boldsymbol{S}^{\mathrm{e}}+\boldsymbol{\alpha}^{\mathrm{tT}} \boldsymbol{E}^{\mathrm{e}}+\boldsymbol{\mu}^{\mathrm{t}} \boldsymbol{H}^{\mathrm{e}},
\end{aligned}
$$

where superscripts e and t mean for the "effective" and "total" respectively, where $\alpha$ is the postulated magnetoelectric coefficient tensor and $\gamma^{\mathrm{T}}$ is the transposed $\gamma$. The magnetoelectric coupling is a new property of the composite. Of these constitutive coefficient tensors, $C$ is fourth-rank tensors; $\boldsymbol{e}\left(\boldsymbol{e}^{\mathrm{t}}\right)$ and $\boldsymbol{q}\left(\boldsymbol{q}^{\mathrm{t}}\right)$ are three-rank tensors; and $\varepsilon$ and $\mu$ and $\alpha\left(\alpha^{\mathrm{t}}\right)$ are second rank tensors. If we obtain some formula for $\alpha$ in such materials, it will prove the existence of the EM coupling in the consideration. The elastic equations of motion of the superlattice can then be written as

$$
\rho \frac{\partial^{2} u_{i}}{\partial t^{2}}-T_{i j, j}=0 \text {. }
$$

Taking into account the Maxwell equation with "polarization currents", we can write

$$
\begin{aligned}
(\boldsymbol{k} \times \boldsymbol{H})_{i} & =\omega\left(\varepsilon_{i k} E_{k}+e_{j k i} \frac{\partial u_{k}}{\partial x_{j}}\right) \theta_{p}, \\
(\boldsymbol{k} \times \boldsymbol{E})_{i} & =\omega\left(\mu_{i k} H_{k}+q_{i j k} \frac{\partial u_{k}}{\partial x_{j}}\right) \theta_{m},
\end{aligned}
$$

with $\varepsilon(z)=\varepsilon_{0}-\delta \varepsilon \theta_{p}(z), \mu(z)=\mu_{0}-\delta \mu \theta_{m}(z)$ From the elastic equation of motion and taking into account (3-6), we obtain

$$
\begin{aligned}
& \rho \frac{\partial^{2} u_{j}}{\partial t^{2}}-C_{i j k l} \frac{\partial^{2} u_{k}}{\partial x_{j} \partial x_{l}}+\frac{\partial\left(e_{k j l}(z) \boldsymbol{E}_{l}\right)}{\partial x_{j}} \\
& +\frac{\partial\left(q_{i j l} \boldsymbol{H}_{l k}\right)}{\partial x_{j}}=0 .
\end{aligned}
$$

By using the Fourier transformation, we have for these 
fields

$$
\begin{aligned}
& u_{j}=\int \tilde{u}_{J} \mathrm{e}^{\mathrm{j}(\omega t-\boldsymbol{q} \boldsymbol{r})} \mathrm{d} \boldsymbol{q}, \\
& (\boldsymbol{E}, \boldsymbol{H})=\int(\tilde{\boldsymbol{E}}, \tilde{\boldsymbol{H}})(\boldsymbol{k}) \mathrm{e}^{\mathrm{j}(\omega t-\boldsymbol{k} \boldsymbol{r})} \mathrm{d} \boldsymbol{k}
\end{aligned}
$$

and for material coefficients

$$
\begin{aligned}
& \boldsymbol{\gamma}=(\rho, \boldsymbol{C}, \boldsymbol{e}, \boldsymbol{q}, \boldsymbol{\varepsilon}, \boldsymbol{\mu}), \\
& \gamma(z)=\sum_{\boldsymbol{K}} \tilde{\gamma}(\boldsymbol{K}) \mathrm{e}^{-\mathrm{j} \boldsymbol{K} \boldsymbol{r}},
\end{aligned}
$$

where $\boldsymbol{q}$ is the elastic wave vector, $\boldsymbol{k}$ is the electromagnetic wave vector, and $\boldsymbol{K}=K \hat{\boldsymbol{z}}$ is the reciprocal vector of the periodic multilayer. Following the way developed in [5] for piezoelectric superlayers the formula (7) is expressed as

$$
\begin{aligned}
& \int\left(-\rho \omega \delta_{j k}+c_{i j k l} q_{i} q_{l}\right) \tilde{u}_{k} \mathrm{e}^{-\mathrm{j} \boldsymbol{q} \boldsymbol{r}} \mathrm{d} \boldsymbol{q} \\
& =-\mathrm{j} \sum_{\boldsymbol{K}}(\boldsymbol{\kappa})_{i}\left[\tilde{e}_{l j n}(\boldsymbol{\kappa}) \tilde{E}_{n}+\tilde{q}_{i l j n}(\boldsymbol{\kappa}) \tilde{H}_{n}\right],
\end{aligned}
$$

where $\boldsymbol{\kappa}=\boldsymbol{K}+\boldsymbol{k}$.

In order for the two sides of (8) to be equal, $\boldsymbol{q}$ must satisfy $\boldsymbol{q}=\boldsymbol{\kappa}$ for one of the $\boldsymbol{K}$ vectors.

For the Bloch-Fourier transformed displacements we can read

$$
\tilde{u}_{k}=-\mathrm{j} G_{j k}(\boldsymbol{\kappa})\left(\tilde{q}_{l j n} \tilde{H}_{n} \tilde{\theta}_{m}+\tilde{e}_{l j n} \tilde{H}_{n} \tilde{\theta}_{p}\right)
$$

with

$$
G_{j k}(\boldsymbol{\kappa})=\left(-\rho \omega^{2} \delta_{j k}+c_{i j k l} \kappa_{i} \kappa_{l}\right)^{-1}
$$

corresponding to the Fourier transformed Green function, and

$$
\frac{\partial u_{k}}{\partial x_{j}}=\mathrm{e}^{-\mathrm{j}(\boldsymbol{k}+\boldsymbol{K}) \boldsymbol{r}} \boldsymbol{\kappa}_{j} \tilde{G}_{i j}(\boldsymbol{\kappa})\left(\tilde{q}_{l j m} \tilde{H}_{m} \tilde{\theta}_{m}+\tilde{e}_{l j n} \tilde{E}_{n} \tilde{\theta}_{p}\right) .
$$

Then

$$
\begin{aligned}
& \left(-\rho \omega^{2} \delta_{j k}+c_{i j k l} \kappa_{i} \kappa_{l}\right) \tilde{u}_{k}(\boldsymbol{\kappa}) \\
& \quad=\mathrm{j} \kappa_{j}\left[\tilde{e}_{l j n}(\boldsymbol{\kappa}) \tilde{E}_{n}+\tilde{q}_{i l j n}(\boldsymbol{\kappa}) \tilde{H}_{n}\right] .
\end{aligned}
$$

The polarization support is known to rather strongly fall towards consecutive boundaries. This is produced by a size effect of homogeneous regions, which is familiar for both ferroelectrics and ferromagnetic monodomain layers (see e.g. [6]).

Thus, it takes rather trapezoidal than a rectangular form. For brevity, we assume here that $z$ cross-section for both (electric and magnetic) layers are identical through the structure having an equilateral trapezoid form, depicted in Fig. 2.

Then the Fourier transform of the assumed trapezoid shape of each layer cross-section takes the form

$$
W_{\text {trop }}(\boldsymbol{\kappa})=\operatorname{Trap}(\boldsymbol{\kappa}) \mathrm{e}^{-\mathrm{j} \frac{\kappa d}{2}}=Q_{p}(\boldsymbol{\kappa})=Q_{m}(\boldsymbol{\kappa}),
$$
where

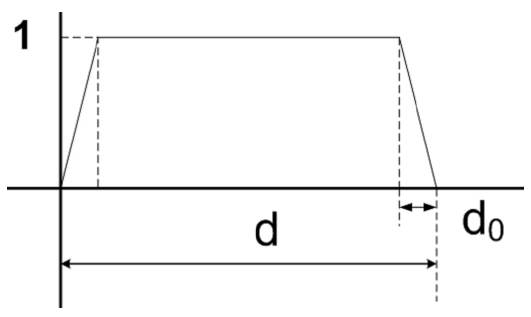

Fig. 2. The model shape of $z$ cross-section for one-layer polarization and magnetization.

$$
\operatorname{Trap}(\kappa)=\frac{4 \sin \left(\frac{d_{0} \kappa}{2}\right)}{\kappa d_{0}} \frac{\sin \left(\frac{\kappa\left(d-d_{0}\right)}{2}\right)}{\kappa} .
$$

For the effective fields in all the structure, we can write

$$
\begin{aligned}
& D_{m}^{\mathrm{e}}=e_{m k l}^{\mathrm{t}} \frac{\partial u_{k}}{\partial x_{l}}+\varepsilon_{m l} E_{l}^{\mathrm{e}}=\varepsilon_{m l}^{\mathrm{t}} E_{l}^{\mathrm{e}}, \\
& B_{i}^{\mathrm{e}}=q_{m k l}^{\mathrm{t}} \frac{\partial u_{k}}{\partial x_{l}}+\mu_{m l} H_{l}^{\mathrm{e}}=\mu_{m l}^{\mathrm{t}} H_{l}^{\mathrm{e}}, \\
& \frac{\partial \tilde{u}_{k}}{\partial x_{l}}=\mathrm{e}^{-\mathrm{j} \boldsymbol{\kappa} \rho} \kappa_{i} \kappa_{l} \tilde{G}_{j k}(\boldsymbol{\kappa})\left(\tilde{e}_{i j n} E_{n}^{\mathrm{t}}+\tilde{q}_{i j n} H_{n}^{\mathrm{t}}\right), \\
& D_{m}^{\mathrm{t}}=e_{m k l}^{\mathrm{T}} \mathrm{j} \mathrm{e}^{\mathrm{j} \boldsymbol{k} \boldsymbol{\rho}+\mathrm{j} \boldsymbol{K} \boldsymbol{z}} \kappa_{l} \tilde{G}_{j k}\left(\tilde{q}_{k j n} H_{n}^{\mathrm{t}}+\tilde{e}_{k j n} E_{n}^{\mathrm{t}}\right) \\
& +\varepsilon_{m n} E_{n} \text {. }
\end{aligned}
$$

We then obtain

$$
\varepsilon_{i k}^{\mathrm{e}}=\varepsilon_{i k}+e_{i k l}^{\mathrm{T}} \mathrm{j} \kappa_{j} \tilde{G}_{i j}(\kappa) \tilde{e}_{l k i j} \mathrm{e}^{\mathrm{j}(\boldsymbol{k} \boldsymbol{\rho}+K z)}
$$

with $\boldsymbol{\rho}=(x, y)$.

$$
\begin{aligned}
& B_{i}^{\mathrm{e}}=q_{m k l}^{\mathrm{T}} \mathrm{j} \mathrm{e}^{\mathrm{j} \boldsymbol{k} \boldsymbol{\rho}+\mathrm{j} \boldsymbol{K} \boldsymbol{z}} \boldsymbol{\kappa}_{l} \tilde{G}_{j k}\left(\tilde{q}_{k j n} H_{n}+\tilde{e}_{k j n} E_{k}\right) \\
& \quad+\mu_{m n} H_{n},
\end{aligned}
$$

as well as

$$
\alpha_{m n}=e_{i j n} \kappa_{l} \kappa_{i} G_{j k}(\boldsymbol{\kappa}) \tilde{q}_{i j n} \theta_{m}(\boldsymbol{\kappa}) \theta_{p}(\boldsymbol{\kappa}) .
$$

Substituting (17) into (15) and (16), we get

$$
\begin{aligned}
& B_{i}=\mu_{i k}(\boldsymbol{\kappa}) \tilde{H}_{k}^{\mathrm{e}}, \\
& D_{i}=\varepsilon_{i k}(\boldsymbol{\kappa}) \tilde{E}_{k}^{\mathrm{e}},
\end{aligned}
$$

where

$$
\begin{aligned}
& \mu_{\mathrm{in}}^{\mathrm{t}}(\boldsymbol{\kappa})=\mu_{\mathrm{in}}^{S}+\tilde{q}_{i k l} \kappa_{l} \tilde{G}_{j k}(\boldsymbol{\kappa}) \kappa_{p} \tilde{q}_{p j n} \theta_{m}(\boldsymbol{\kappa}), \\
& \varepsilon_{i k}^{\mathrm{t}}=\varepsilon_{i k}^{S}+\tilde{e}_{i k l} \kappa_{l} \tilde{G}_{j k}(\boldsymbol{\kappa}) \kappa_{p} \tilde{e}_{p j n} \theta_{p}(\boldsymbol{\kappa}),
\end{aligned}
$$

together with (21), which provides the effective magnetoelectric dynamic coupling coefficient.

In the formulae above the Einstein summation convention is applied.

\section{Concluding remarks}

The resulting formulae are expressed in the simple analytical closed form which clearly illustrates the mechanism of the dynamic magnetoelectric coupling, as mediated by the elastic strain wave. It is instructive to note 
that all the far field interaction is effectively described by the nonlocal elastic Green function, which effectively extends over the entire composite sample.

The three principal effective module tensors, i.e., the effective elastic stiffness $\boldsymbol{C}^{\mathrm{t}}$, the effective dielectric permittivity tensor $\varepsilon^{\mathrm{t}}$, and the effective magnetic permeability $\mu^{\mathrm{t}}$, all contain the coupled magnetic-electrical-mechanical effect.

The effective dielectric tensor $\varepsilon^{t}$, in (24), contains also the piezomagnetic effect. If the piezomagnetic effect is ignored, the formula (24) becomes the known result for piezoelectric composites. The effective piezomagnetic tensor $\boldsymbol{q}^{\mathrm{t}}$ is similar to the effective piezoelectric tensor $\boldsymbol{e}^{\mathrm{e}}$. The behavior of $\boldsymbol{q}^{\mathrm{t}}$ for the piezomagnetic composites is similar to that of $\boldsymbol{e}^{\mathrm{t}}$ for the piezoelectric composites.

Equation (21) for the effective magnetoelectric coefficient tensor $\alpha$ expressing the piezoelectric-piezomagnetic coupling is an important result. Similarly to the static case investigated e.g. by Nan et al. [1-3], we see that this is also the product property of piezoelectric and piezomagnetic effects this time via the dynamic elastic coupling. The composite, indeed, has a nonzero dynamic magnetoelectric effect, though such effect is absent in the two constituent phases.

It is of importance to notice that apart from the strain mediated coupling mechanism, there are also an- other mechanisms responsible for magnetoelectric coupling. Especially important is the interface charge coupling which appears within the boundary of particular layers, being a "depletion of polarization" as shown in Fig. 2. It has been shown, however, [7-9], that this effect is the main ME coupling mechanism only when the composite film thickness is below certain transition thickness $d_{\mathrm{tr}}$ while the strain-mediated coupling dominate above $d_{\mathrm{tr}}$.

\section{References}

[1] C.-W. Nan, Phys. Rev. B 50, 6082 (1994).

[2] C.W. Nan, M.I. Bichurin, S. Dong, D. Viehland, G. Srinivasan, J. Appl. Phys. 103, 31101 (2008).

[3] G. Srinivasan, Anu. Rev. Mater. Res. 40, 153 (2010).

[4] V.G. Kukhar, Nanotechnology 21, 265701 (2010).

[5] H. Liu, Phys. Rev. B 71, 125106 (2005).

[6] R. Kretschmer, K. Binder, Phys. Rev. B 20, 1065 (1979).

[7] J.-M. Hu, C.W. Nan, Phys. Rev. B 80, 224416 (2009).

[8] C.A.F. Vaz, Phys. Rev. Lett. 104, 127202 (2010).

[9] M. Endo, Appl. Phys. Lett. 96, 212503 (2010). 